\title{
STATIONARY SETS OF THE MEAN CURVATURE FLOW WITH A FORCING TERM
}

\author{
VESA JULIN AND JOONAS NIINIKOSKI
}

\begin{abstract}
We consider the flat flow solution to the mean curvature equation with forcing in $\mathbb{R}^{n}$. Our main results states that tangential balls in $\mathbb{R}^{n}$ under a flat flow with a bounded forcing term will experience fattening, which generalizes the result in [11] from the planar case to higher dimensions. Then, as in the planar case, we characterize stationary sets in $\mathbb{R}^{n}$ for a constant forcing term as finite unions of equisize balls with mutually positive distance.
\end{abstract}

\section{INTRODUCTION}

In this article we consider the mean curvature flow (MCF) with a bounded forcing term for compact embedded hypersurfaces. By definition this is a family of embedded surfaces $\left(\Sigma_{t}\right)_{t \in[0, \infty)}$ in $\mathbb{R}^{n}$, with initial set $\Sigma_{0}$, and which moves according to the law

$$
V_{t}=-H_{\Sigma_{t}}+f(t)
$$

where $V_{t}$ is the normal velocity, $H_{\Sigma_{t}}$ the mean curvature and $f$ a bounded measurable function. It is well known that the flow may develop singularities for a smooth initial set when $n \geq 3$ [12] and even in the plane when $f \neq 0$ [4]. In order to define the flow over the singular times and in order to define it for rough initial sets, one may define a weak solution by using either the level set formulation [7, 10], the flat flow via the minimizing movement scheme $[1,14]$ or Brakke's varifold formulation [5]. The main issue is that there is no unique way to define the weak solution and the previous methods may give rise to a different solution. The level-set approach provides a unique function which is a solution of the corresponding partial differential equation in the viscosity sense, but its level sets may have positive volume. We call this phenomenon fattening. De Giorgi's minimal and maximal barriers provide essentially the same solution as the level-set approach and in this context the fattening means that the minimal and the maximal solution do not agree. The fattening may occur instantaneously if the initial set is not regular $[4,10]$ or after a finite time for regular initial sets [4]. In this work we consider the flat flow of (1.1), which is a solution obtained via the minimizing movement scheme as in $[1,14]$. The flat flow can be defined for rough embedded initial hypersurfaces which are boundaries of sets of finite perimeter. Therefore, it is more natural in this context to define the flow for sets rather than surfaces. If the initial set is smooth the flat flow agrees with the classical solution for a short time interval, but in case of fattening it is not clear if it is uniquely defined.

Here we study the fattening for the flat flow of (1.1) in the specific case when the initial set is a union of two tangent balls. It is well known that in this case the level-set solution produces instantaneously fattening $[4,13]$. We also mention the work [9] where the authors study the same setting but add randomness to the flow. For a general introduction to the topic we refer to [3]. In our main theorem we generalize the result in [11] from the plane to 
$\mathbb{R}^{n}$ and prove that the flat flow instantaneously connects the two tangent balls with a thin neck which continues to grow at least for a short period of time.

Theorem 1.1. Let $E_{0} \subset \mathbb{R}^{n}, n \geq 2$, be a union of two tangential balls $B\left(x_{1}, r\right)$ and $B\left(x_{2}, r\right)$. Let $\left(E_{t}\right)_{t}$ be a flat flow with forcing term $f$, which is bounded by $C_{0} \in \mathbb{R}_{+}$, starting from $E_{0}$. There exist positive numbers $\delta, c_{1}$ and $c_{2}$ depending only on $n, r$ and $C_{0}$ such that for every $t \in(0, \delta)$ the set $E_{t}$ contains a dumbbell shaped simply connected set which again contains the balls $B\left(x_{1}, r-c_{1} t\right), B\left(x_{2}, r-c_{1} t\right)$ and $B\left(\left(x_{1}+x_{2}\right) / 2, c_{2} t\right)$.

We note that the above result immediately generalizes to the case when the two balls do not have the same radii. This follows from Theorem 1.1 and a standard comparison argument (see Proposition 3.2).

Theorem 1.1 implies that a union of tangent balls cannot be a stationary set of the flow (1.1). Therefore, we may use the characterization of critical points of the isoperimetric problem from [8] to characterize all stationary points of the flow (1.1).

Theorem 1.2. A bounded set of finite perimeter $E_{0} \subset \mathbb{R}^{n}$, with $n \geq 2$, is a stationary set of the flow (1.1) (see definition 3.5) with a positive constant forcing $\Lambda$ exactly when it is a finite union of balls of radius $r=(n-1) / \Lambda$ with mutually positive distance.

Let us finally mention a few words about the proof of Theorem 1.1. We begin the proof as in the planar case [11] by showing that any discrete approximation of the flat flow creates at the first step a neck which connects the two balls. After this we need to show that this neck is growing until the time $\delta$. In the planar case it is enough to construct a single barrier set to show that the neck is growing (see [11, Proof of Theorem 1.1]). In the higher dimensional case we need to construct a family of comparison sets which, together with a delicate comparison argument, implies that the neck is growing. The novelty of the proof is the construction of this discrete barrier flow. Similar idea is used in [13] in the context of level set solutions. The main difference is that in our case the flow is defined via time discretization.

\section{Notation AND PRELIMINARY RESUlts}

Let us introduce some basic concepts and notation. First, our standing assumption throughout the paper is that the dimension $n$ is at least two and for $x \in \mathbb{R}^{n}$ we use the decomposition $x=\left(x_{1}, x^{\prime}\right)$, where $x_{1} \in \mathbb{R}$ and $x^{\prime} \in \mathbb{R}^{n-1}$. For a given set $E \subset \mathbb{R}^{n}$ the distance function $d_{E}: \mathbb{R}^{n} \rightarrow \mathbb{R}$ is given by $d_{E}(x)=\inf _{y \in E}|x-y|$ and further the signed distance function $\bar{d}_{E}: \mathbb{R}^{n} \rightarrow \mathbb{R}$ is defined as

$$
\bar{d}_{E}(x)= \begin{cases}-d_{E}(x), & x \in E \\ d_{E}(x), & x \in \mathbb{R}^{n} \backslash E .\end{cases}
$$

For the empty set we use the convention that its signed distance function is $\infty$ everywhere. If $E \subset \mathbb{R}^{n}$ is Lebesgue-measurable, we will denote its $n$-dimensional Lebesgue-measure by $|E|$.

For a set of finite perimeter $E \subset \mathbb{R}^{n}$ the notation $\partial^{*} E$ denotes its reduced boundary as usual. Recall, that then $\overline{\partial^{*} E}$ is the support of the corresponding Gauss-Green measure and the perimeter of $E$ is given by $P(E)=\mathcal{H}^{n-1}\left(\partial^{*} E\right)$. If $E$ is $C^{1}$-regular we have $\partial^{*} E=\partial E$. Moreover, we may always assume $\partial E=\overline{\partial^{*} E}$. The measure theoretic outer unit normal is defined in $\partial^{*} E$ and we denote it by $\nu_{E}$. If $E$ is a $C^{1}$-set, then $\nu_{E}$ agrees with the classical outer unit normal of $E$. Again, for every $C^{1}$-vector field $\Psi: \mathbb{R}^{n} \rightarrow \mathbb{R}^{n}$ the tangential differential at $x$ is defined as

$$
\mathrm{D}_{\tau} \Psi(x)=\mathrm{D} \Psi(x)\left(I-\nu_{E}(x) \otimes \nu_{E}(x)\right)
$$


and the tangential divergence as $\operatorname{div}_{\tau} \Psi=\operatorname{Tr}\left(\mathrm{D}_{\tau} \Psi(x)\right)$.

For an orientable $C^{2}$-hypersurface $\Sigma \subset \mathbb{R}^{n}$, with orientation $\nu_{\Sigma}: \Sigma \rightarrow \partial B(0,1)$, the corresponding mean curvature $H_{\Sigma}(x)$ at $x \in \Sigma$ is defined as the sum of the principal curvatures $k_{1}(x), \ldots, k_{n-1}(x)$. If $E \subset \mathbb{R}^{n}$ is a $C^{2}$-set, then $H_{E}(x)$ for $x \in \partial E$ denotes $H_{\partial E}(x)$, with the orientation $\nu_{E}$, and we have the classical (surface) divergence theorem

$$
\int_{\partial E} \operatorname{div}_{\tau} \Psi \mathrm{d} \mathcal{H}^{n-1}=\int_{\partial E} H_{E}\left\langle\Psi, \nu_{E}\right\rangle \mathrm{d} \mathcal{H}^{n-1}
$$

for every $\Psi \in C_{0}^{1}\left(\mathbb{R}^{n} ; \mathbb{R}^{n}\right)$. In general, we say that a set of finite perimeter $E \subset \mathbb{R}^{n}$ has a distributional mean curvature $H_{E} \in L^{1}\left(\partial^{*} E\right)$, if for every $\Psi \in C_{0}^{1}\left(\mathbb{R}^{n} ; \mathbb{R}^{n}\right)$ it holds

$$
\int_{\partial^{*} E} \operatorname{div}_{\tau} \Psi \mathrm{d} \mathcal{H}^{n-1}=\int_{\partial^{*} E} H_{E}\left\langle\Psi, \nu_{E}\right\rangle \mathrm{d} \mathcal{H}^{n-1} .
$$

Note that for $C^{2}$-regular sets the distributional mean curvature agrees with the classical mean curvature. Finally, we say that a set of finite perimeter $E \subset \mathbb{R}^{n}$ is critical, if it has a constant distributional mean curvature. By [8, Theorem 1] we know that the critical sets are characterized as finite union of balls with equal radius and mutually disjoint interiors. As a consequence, we have the following convergence result, see [8, Corollary 2].

Theorem 2.1. Let $\left(E_{i}\right)_{i=1}^{\infty}$ be a sequence of sets of finite perimeters in $\mathbb{R}^{n}$ with distributional mean curvature $H_{E_{i}}, E \subset \mathbb{R}^{n}$ a set of finite perimeter with a positive volume and $\Lambda$ a positive constant such that $\left|E \Delta E_{i}\right| \rightarrow 0, P\left(E_{i}\right) \rightarrow P(E)$ and $H_{E_{i}} \rightarrow \Lambda$ in the distributional sense, i.e., for every $\Psi \in C_{0}^{1}\left(\mathbb{R}^{n} ; \mathbb{R}^{n}\right)$ it holds

$$
\lim _{i \rightarrow \infty} \int_{\partial^{*} E_{i}} \operatorname{div}_{\tau} \Psi-\Lambda\left\langle\Psi, \nu_{E_{i}}\right\rangle \mathrm{d} \mathcal{H}^{n-1}=0 .
$$

Then $E$ is a finite union of balls with the equal radius $r=(n-1) / \Lambda$ and the balls have mutually disjoint interiors.

We will use solid of revolutions which are obtained by rotating a non-negative function around the $x_{1}$-axis in $\mathbb{R}^{n}$. If $g$ is a non-negative function defined on an interval $[a, b]$, we will denote by $\mathbf{C}(g,[a, b])$ the solid of revolution

$$
\mathbf{C}(g,[a, b]):=\left\{x \in \mathbb{R}^{n}: x_{1} \in[a, b], x^{\prime} \in \bar{B}^{n-1}\left(0, g\left(x_{1}\right)\right)\right\} .
$$

Again, by the heads of $\mathbf{C}(g,[a, b])$ we mean the vertical part of the boundary

$$
\left\{x \in \mathbb{R}^{n}: x_{1} \in\{a, b\}, x^{\prime} \in \bar{B}^{n-1}\left(0, g\left(x_{1}\right)\right)\right\} .
$$

In the special case of a cylinder, symmetric to the hyperplane $\left\{x_{1}=0\right\}$, i.e., $g \equiv R>0$ and $b=-a$, we simply denote $\mathbf{C}(R, a)=\mathbf{C}(R,[-a, a])$. In the case, where $g$ is continuous on $[a, b]$ and vanishes at the endpoints, we make the following technical observation.

Remark 2.2. Suppose that $g \in C([a, b])$ is non-negative with $g(a)=0=g(b)$ and denote $E=\mathbf{C}(g,[a, b])$. Then for every $x_{1} \in \mathbb{R}$ the section $\bar{d}_{E}\left(x_{1}, \cdot\right): \mathbb{R}^{n-1} \rightarrow \mathbb{R}$ is radially symmetric function strictly increasing in radius.

If $g \in C([a, b]) \cap C^{2}((a, b))$ and is strictly positive, then for the surface of revolution

$$
\Gamma=\left\{\left(x_{1}, x^{\prime}\right) \in \mathbb{R}^{n}: x_{1} \in(a, b), x^{\prime} \in \partial B^{n-1}\left(0, g\left(x_{1}\right)\right)\right\}
$$


with the inside-out orientation of $\mathbf{C}(g,[a, b])$ one computes

$$
H_{\Gamma}(x)=-\frac{g^{\prime \prime}\left(x_{1}\right)}{\left(1+g^{\prime}\left(x_{1}\right)^{2}\right)^{\frac{3}{2}}}+\frac{1}{\left(1+g^{\prime}\left(x_{1}\right)^{2}\right)^{\frac{1}{2}}} \frac{n-2}{g\left(x_{1}\right)}
$$

for every $x \in \Gamma$.

A solid of revolution $\mathbf{C}(g,[a, b])$ is an example of a Schwarz symmetric set. Recall, that for every measurable set $E \subset \mathbb{R}^{n}$ its Schwarz symmetrization, or $(n-1)$-dimensional Steiner symmetrization, with respect to a direction $e \in \partial B(0,1)$ is a measurable set $E_{e}^{*}$ such that for every $t \in \mathbb{R}$ the section $\left\{z \in\langle e\rangle^{\perp}: t e+z \in E_{e}^{*}\right\}$ is an open $(n-1)$-dimensional ball centered at the origin and it holds

$$
\mathcal{H}^{n-1}\left(\left\{z \in\langle e\rangle^{\perp}: t e+z \in E\right\}\right)=\mathcal{H}^{n-1}\left(\left\{z \in\langle e\rangle^{\perp}: t e+z \in E_{e}^{*}\right\}\right) .
$$

Note that $\left|E_{e}^{*}\right|=|E|$ and if $E$ is a set of finite perimeter, then $E_{e}^{*}$ is also a set of finite perimeter and $P\left(E_{e}^{*}\right) \leq P(E)[2]$. A set $E$ is Schwarz symmetric with respect to $e$ if it holds $E_{e}^{*}=E$, up to a set of measure zero.

\section{Flat FLOWS With FORCING AND STATIONARY SETS}

Let us first heuristically explain how a flat flow with a forcing term is obtained via the minimizing movement scheme. Let $C_{0} \in \mathbb{R}_{+}$be a fixed constant and let $f:[0, \infty) \rightarrow \mathbb{R}$ be a measurable function satisfying the condition

$$
\sup _{t \geq 0}|f(t)| \leq C_{0} .
$$

The function $f$ will act as a time dependent forcing term in the dynamics. Now if $E_{0}$ is a bounded set of finite perimeter, we define for every $0<h \leq 1$ a sequence of a bounded sets of finite perimeter $\left(E^{h, k}\right)_{k=0}^{\infty}$, so called approximative sequence, inductively by setting first $E^{h, 0}=E_{0}$ and for $k=0,1,2, \ldots$ we set $E^{h, k+1}$ to be a minimizer of the functional

$$
F \mapsto P(F)+\frac{1}{h} \int_{F} \bar{d}_{E^{h, k}} \mathrm{~d} x-\bar{f}(h, k)|F|,
$$

where $\bar{f}(h, k)=f_{k h}^{(k+1) h} f(t) \mathrm{d} t$. Then we define an approximate flat flow $\left(E_{t}^{h}\right)_{t \geq 0}$ by setting

$$
E_{t}^{h}=E^{h, k} \text { for } k h \leq t<(k+1) h .
$$

If there is a subsequence $\left(h_{k}\right)_{k \in \mathbb{N}}$ with $h_{k} \rightarrow 0$ and a family of bounded sets of finite perimeter $\left(E_{t}\right)_{t \geq 0}$ such that $E^{h_{k}} \rightarrow E_{t}$ for every $t \geq 0$ in the $L^{1}$-sense, then we call $\left(E_{t}\right)_{t \geq 0}$ a flat flow with forcing $f$ starting from $E_{0}$. An existence of such a cluster point is always guaranteed, see for instance [11, Proposition 2.3].

Let us next make the above argument more precise by using the results in $[11,16]$. We note that in [16] the authors consider flat flow for volume preserving mean curvature flow, but the arguments will remain valid in our setting. Our first observation is that the functional in (3.2) may change its values if we perturb the set $E^{h, k}$ by a set of measure zero due to the distance function. In order to use the notion of distance function consistently we define the class

$$
X_{n}=\left\{E \subset \mathbb{R}^{n}: E \text { is a bounded set of finite perimeter with } \partial E=\overline{\partial^{*} E}\right\} .
$$

Recall, that every (essentially) bounded set of finite perimeter has a $L^{1}$-equivalent set from $X_{n}$. For given $0<h \leq 1$ and $\Lambda \in\left[-C_{0}, C_{0}\right]$ we define the functional

$$
\mathcal{F}_{h, \Lambda}: X_{n} \times X_{n} \rightarrow \mathbb{R} \cup\{\infty\}
$$


by setting

$$
\mathcal{F}_{h, \Lambda}(F, E)=P(F)+\frac{1}{h} \int_{F} \bar{d}_{E} \mathrm{~d} x-\Lambda|F| .
$$

For every $E \in X_{n}$ the functional $\mathcal{F}_{h, \Lambda}(\cdot, E)$ admits a minimizer $E_{\min } \in X_{n}$, see [16, proof of Lemma 3.1]. If $E$ is empty, then $\bar{d}_{E}=\infty$ and hence necessarily $E_{\min }$ must be empty too. Minimizers have the following distance property, see the proof of [14, Lemma 2.1] (or [16, Proposition 3.2]). There is a positive constant $\gamma=\gamma\left(n, C_{0}\right)$ such that for every $E \in X_{n}$ and every minimizer $E_{\min } \in X_{n}$ of $\mathcal{F}_{h, \Lambda}(\cdot, E)$ it holds

$$
\left|\bar{d}_{E}\right| \leq \gamma h^{\frac{1}{2}} \text { in } E \Delta E_{\min } .
$$

Now, (3.5) has the following consequence.

Remark 3.1. Suppose that $E_{1}, E_{2}, \ldots, E_{k} \in X_{n}$ have a mutually positive distance of at least of $d$. There is a positive $h_{d}=h_{d}\left(n, C_{0}, d\right) \leq 1$ such that for any $h \leq h_{d}$ it holds that any minimizer of $\mathcal{F}_{h, \Lambda}\left(\cdot, \bigcup_{i} E_{i}\right)$ must be a union of minimizers of $\mathcal{F}_{h, \Lambda}\left(\cdot, E_{i}\right)$.

In general, uniqueness of a minimizer of $\mathcal{F}_{h, \Lambda}(\cdot, E)$ is not known. However, the following weak comparison principle holds, see [6, proof of Lemma 7.2].

Proposition 3.2. Let $E, E^{\prime} \in X_{n}$ and $\Lambda, \Lambda^{\prime} \in\left[-C_{0}, C_{0}\right]$, with $\Lambda>\Lambda^{\prime}$.

(i) If $E^{\prime} \subset \subset E$ and $E_{\min }, E_{\min }^{\prime} \in X_{n}$ are minimizers of $\mathcal{F}_{h, \Lambda}(\cdot, E)$ and $\mathcal{F}_{h, \Lambda}\left(\cdot, E^{\prime}\right)$ respectively, then $\left|E_{\min }^{\prime} \backslash E_{\min }\right|=0$.

(ii) If $E^{\prime} \subset E$ and $E_{\min }, E_{\min }^{\prime} \in X_{n}$ are minimizers of $\mathcal{F}_{h, \Lambda}(\cdot, E)$ and $\mathcal{F}_{h, \Lambda^{\prime}}\left(\cdot, E^{\prime}\right)$ respectively, then $\left|E_{\min }^{\prime} \backslash E_{\min }\right|=0$.

Concerning the regularity of a minimizer $E_{\min }$ of (3.4), it is not difficult to see that it is a $\left(\Lambda_{0}, r_{0}\right)$-perimeter minimizer (using the notation from [15]) with suitable $\Lambda_{0}, r_{0} \in \mathbb{R}_{+}$ satisfying $\Lambda_{0} r_{0} \leq 1$. Then it follows [15, Theorem 26.5 and Theorem 28.1] that $\partial^{*} E_{\min }$ is relatively open in $\partial E_{\min }, C^{1, \alpha}$-regular hypersurface for every $0<\alpha<1 / 2$ and the (closed) singular part $\partial E \backslash \partial^{*} E$ has Hausdorff-dimension at most $n-8$. In particular, from now on we will use the convention that the minimizers are always open sets.

Moreover, by considering local variations $\left(\Phi_{t}\right)_{t}$ of the form $\Phi_{t}=\mathrm{id}+t \Psi$, with $\Psi \in C_{0}^{1}\left(\mathbb{R}^{n}, \mathbb{R}^{n}\right)$, and differentiating $t \mapsto \mathcal{F}_{h, \Lambda}\left(\Phi_{t}\left(E_{\min }\right), E\right)$ at zero we see that $E_{\min }$ has distributional mean curvature $H_{E_{\min }}$ which satisfies the Euler-Lagrange equation in the distributional sense

$$
\frac{\bar{d}_{E}}{h}=-H_{E_{\min }}+\Lambda \text { on } \partial^{*} E_{\min } .
$$

Since $H_{E_{\min }}$ is Lipschitz continuous on $\partial^{*} E$, then by standard elliptic estimates $\partial^{*} E_{\min }$ is $C^{2, \alpha}$-regular and (3.6) holds in the classical sense on the reduced boundary. In particular, $E_{\min }$ is a $C^{2, \alpha}$-set when $n \leq 7$. Finally, we note that if $\partial E$ satisfies an exterior or interior ball condition at $x$, then $x$ must belong to the regular part $\partial^{*} E_{\min }$. This follows essentially from [8, Lemma 3].

The next proposition states the somewhat obvious fact that for a ball $E=B(x, r)$ any non-empty minimizer of $\mathcal{F}_{h, \Lambda}(\cdot, E)$ must be a concentric ball.

Proposition 3.3. For a ball $E=B(x, r)$ every minimizer (3.4) must be an open concentric ball or the empty set. There is a positive constant $h_{0}=h_{0}\left(n, C_{0}\right) \leq 1$ such that if $h \leq h_{0}$, then 
every ball $B(x, r)$, with $r \geq(n-1) / C_{0}$, has a concentric ball $B\left(x, r_{\min }\right)$ as a unique minimizer of $\mathcal{F}_{h, \Lambda}(\cdot, E)$ and it holds

$$
r_{\min }-r=\left[\Lambda-\frac{n-1}{r}+\mathcal{O}(h)\right] h .
$$

In the case $\Lambda=(n-1) / r$, the error term $\mathcal{O}(h)$ vanishes and hence $r_{\min }=r$.

Proof. The first claim is easy to see by using the isoperimetric inequality and the fact that for a given non-zero volume $V$ an open ball of the volume $V$, centered at $x$, is a unique minimizer of the energy $\int_{F} \bar{d}_{B(x, r)} \mathrm{d} y$ among the open sets $F$ of the volume $V$. Again, by using (3.5) we see that if $h$ is sufficiently small compared to the radius, then every minimizer must be non-empty and hence a concentric ball. Thus, the uniqueness and (3.7) follow from (3.5) and the Euler-Lagrange equation (3.6).

Let us denote the Schwarz symmetrization of $E$ with respect to $x_{1}$-axis simply by $E^{*}$. As we mentioned above, Schwarz symmetrization decreases the perimeter and preserves the volume. Moreover, for a smooth set in the case of equality $P\left(E^{*}\right)=P(E)$ it holds that every vertical slice $E_{x_{1}}=\left\{x^{\prime} \in \mathbb{R}^{n-1}:\left(x_{1}, x^{\prime}\right) \in E\right\}$ is $(n-1)$-dimensional ball [2]. We also notice that if the set $E$ is Schwarz symmetric with respect to $x_{1}$-axis, then Schwarz symmetrization also decreases the dissipation term of $\mathcal{F}_{h, \Lambda}(\cdot, E)$ defined in (3.4). This follows rather directly from Fubini's theorem. For a suitable solid of revolution $E$ around the $x_{1}$-axis, there is invariance of minimizers under the symmetrization.

Proposition 3.4. If $E=\mathbf{C}(g,[a, b])$, with a non-negative and continuous $g$ attaining the zero value at the endpoints, then every (open) minimizer $F$ of $\mathcal{F}_{h, \Lambda}(\cdot, E)$ defined in (3.4) is Schwarz symmetric with respect to $x_{1}$-axis.

Proof. Let $F$ be a such a minimizer. We may assume $F$ to be non-empty. Now $P\left(F^{*}\right) \leq P(F)$, $\left|F^{*}\right|=|F|$. By Remark 2.2 every section $\bar{d}_{E}\left(x_{1}, \cdot\right)$ is radially symmetric and strictly increasing in radius which implies via Fubini's theorem that the $(n-1)$-dimensional Lebesgue measure of the symmetric difference $\left|\left(F^{*}\right)_{x_{1}} \Delta F_{x_{1}}\right|_{n-1}$ of the vertical slices $\left(F^{*}\right)_{x_{1}}$ and $F_{x_{1}}$ is zero for almost every $x_{1}$, since otherwise it would hold

$$
\int_{F^{*}} \bar{d}_{E} \mathrm{~d} x<\int_{F} \bar{d}_{E} \mathrm{~d} x
$$

and hence $\mathcal{F}_{h, \Lambda}\left(F^{*}, E\right)<\mathcal{F}_{h, \Lambda}(F, E)$ contradicting the minimality of $F$. Since $F$ is open, then every vertical slice $F_{x_{1}} \subset \mathbb{R}^{n-1}$ is open too and then the previous observation quarantees that $\left(F^{*}\right)_{x_{1}}=F_{x_{1}}$ for almost every $x_{1}$. Thus, the openess of $F$ implies that the equality holds for every $x_{1}$.

After this discussion we are convinced that an approximative sequence $\left(E^{h, k}\right)_{k=0}^{\infty}$, starting from $E_{0}$, where for every $k=1,2 \ldots$, the set $E^{h, k+1}$ is defined as a minimimizer of the functional $\mathcal{F}_{h, \bar{f}(h, k)}\left(\cdot, E^{h, k}\right)$ defined in (3.4), is well-defined. Further, we may define the approximative flat flow $\left(E_{t}^{h}\right)_{t \geq 0}$ as in (3.3). We have for every $t \geq h$ that the set $E_{t}^{h}$ is open and $C^{2}$-regular up to a singular part $\partial E_{t}^{h} \backslash \partial^{*} E_{t}^{h}$ of Hausdorff-dimesion at most $n-8$. Moreover, $E_{t}^{h}$, with $t \geq h$, has a distributional mean curvature $H_{E_{h}^{t}}$ which satisfies the Euler-Lagrange equation (3.6), with $\Lambda=\bar{f}(h,\lfloor t / h\rfloor-1)$, in a weak sense and on $\partial^{*} E_{t}^{h}$ in the classical sense. For more properties of the approximative flat flows, when the forcing term satisfies (3.1), such as local Hölder continuity of $(t, s) \mapsto\left|E_{t}^{h} \Delta E_{s}^{h}\right|$ and perimeter control we refer to [11, Proposition 2.3]. 
Next, we define stationary sets of (1.1) with constant forcing term by using flat flows as in [11, Definition 3.1].

Definition 3.5. A non-empty set $E_{0} \in X_{n}$ is a stationary set of (1.1) for a constant forcing term $f \equiv \Lambda>0$, if for any flat flow, starting from $E_{0}$ it holds

$$
\sup _{0 \leq t \leq T}\left|E_{t} \Delta E_{0}\right|=0
$$

for every $T>0$.

By using Remark 3.1 and Proposition 3.3 one may conclude the obvious direction of Theorem 1.2, that is, a finite union of equisize balls with a mutually positive distance is a stationary set for the constant forcing term $\Lambda=(n-1) / r$, where $r$ is the radius of the balls. In turn, the following lemma states that the converse is almost true, that is, a stationary set is also critical, i.e., a finite union of balls with equal radius and mutually disjoint interiors.

Lemma 3.6. Every stationary set $E_{0} \subset \mathbb{R}^{n}$ for a positive constant forcing term $\Lambda$, is a finite union of balls of radius $r=(n-1) / \Lambda$ with mutually disjoint interiors.

Proof. The lemma is already established in the two-dimensional case in [11, Lemma 3.4]. Again, the proof of the general case is analogous to the proof of [11, Lemma 3.4] with the only essential change is that we use Theorem 2.1 instead of [11, Lemma 3.2]. Therefore, we only sketch the proof. Besides Theorem 2.1, we also use some basic properties of approximate flat flows proven in [11].

We begin by fixing times $0<T_{1}<T_{2}$. Then by Definition 3.5 and [11, Proposition 2.3] we have a decreasing sequence $\left(h_{i}\right)_{i=1}^{\infty}$, with $0<h_{i}<1$ and $h_{i}$ converging to zero, such that the approximate flat flows $\left(E_{t}^{h_{i}}\right)_{t \geq 0}$, with constant forcing $f \equiv \Lambda$ and starting from $E_{0}$ satisfy

$$
\lim _{i \rightarrow \infty} \sup _{t \in\left[T_{1}, T_{2}\right]}\left|E_{0} \Delta E_{t}^{h_{i}}\right|=0 .
$$

Moreover, since the forcing term is constant, it follows from the argument in the proof of [11, Proposition 2.4] that there is $C \in \mathbb{R}_{+}$, independent of $h$, such that for every $t \in\left[T_{1}, T_{2}\right]$ with $t>h$ it holds

$$
\int_{h}^{t} \int_{\partial^{*} E_{s}^{h}}\left|H_{E_{s}^{h}}-\Lambda\right|^{2} \mathrm{~d} \mathcal{H}^{n-1} \mathrm{~d} s \leq C\left[\left(P\left(E_{0}\right)-P\left(E_{t}^{h}\right)\right)+\left(\left|E_{t}^{h}\right|-\left|E_{0}\right|\right)\right] .
$$

Now (3.8) and (3.9) imply limsup $\sup _{i \rightarrow \infty} P\left(E_{t}^{h_{i}}\right) \leq P\left(E_{0}\right)$, for every $t \in\left[T_{1}, T_{2}\right]$. On the other hand, by [11, Proposition 2.3] there is a radius $R>0$, independent of $i$, such that $E_{t}^{h_{i}} \subset$ $B(0, R)$ for every $t \in\left[0, T_{2}\right]$. Then by the lower semi-continuity of the perimeter and by the previous estimate we have $P\left(E_{t}^{h_{i}}\right) \rightarrow P\left(E_{0}\right)$ for every $t \in\left[T_{1}, T_{2}\right]$. Thus, (3.9) yields

$$
\lim _{i \rightarrow \infty} \int_{T_{1}}^{T_{2}} \int_{\partial^{*} E_{t}^{h_{i}}}\left|H_{E_{t}^{h_{i}}}-\Lambda\right|^{2} \mathrm{~d} \mathcal{H}^{n-1} \mathrm{~d} t=0
$$

and further, by the mean value theorem, we find times $t_{i} \in\left(T_{1}, T_{2}\right)$ such that

$$
\lim _{i \rightarrow \infty} \int_{\partial^{*} E_{t_{i}}^{h_{i}}} \mid H_{E_{t_{i}}^{h_{i}}-\left.\Lambda\right|^{2}} \mathrm{~d} \mathcal{H}^{n-1}=0 .
$$

Since $P\left(E_{t_{i}}^{h_{i}}\right)$ are uniformly bounded, we deduce by the previous estimate that $H_{E_{t_{i}}^{h_{i}}} \rightarrow \Lambda$ in the distributional sense. We have also $\left|E_{0} \Delta E_{t_{i}}^{h_{i}}\right| \rightarrow 0$ and $P\left(E_{t_{i}}^{h_{i}}\right) \rightarrow P\left(E_{0}\right)$ so the claim follows from Theorem 2.1. 
Now, the non-trivial direction of Theorem 1.2 is a rather straightforward consequence of Lemma 3.6 and Theorem 1.1, since the latter guarantees that a critical set having two tangential balls cannot be stationary. The reasoning is exactly same as in the planar case, but for the sake of completeness we sketch the argument here. To this aim, let $E_{0}$ be a finite union of balls with equal radius $r$ containing a union of two tangential balls, say

$$
E_{0}^{\prime}=B\left(x_{1}, r\right) \cup B\left(x_{2}, r\right) .
$$

Let $\left(E_{t}\right)_{t \geq 0}$ be any flat flow with a bounded forcing $f$ starting from $E_{0}$. By applying the second claim of Proposition 3.2 we find a a flat flow $\left(E_{t}^{\prime}\right)_{t \geq 0}$ with the forcing $f-1$ starting from $E_{0}^{\prime}$ such that $\left|E_{t}^{\prime} \backslash E_{t}\right|=0$ for every $t \geq 0$. By Theorem 1.1 we have $\left|B\left(\left(x_{1}+x_{2}\right) / 2, c t\right) \backslash E_{t}^{\prime}\right|=0$, and thus $\left|B\left(\left(x_{1}+x_{2}\right) / 2, c t\right) \backslash E_{t}\right|=0$ for some $c \in \mathbb{R}_{+}$and for all small $t>0$. On the other hand, it clearly holds $\left|B\left(\left(x_{1}+x_{2}\right) / 2, c t\right) \backslash E_{0}\right|>0$. Therefore, we deduce that $\left|E_{0} \Delta E_{t}\right|>0$ for all small $t>0$. Thus, $E_{0}$ cannot be stationary.

\section{Proof of Theorem 1.1}

Proof of Theorem 1.1. Let $E_{0}$ be a union of two tangential balls of radius $r$. We may assume that $E_{0}=B\left(-r e_{1}, r\right) \cup B\left(r e_{1}, r\right)$. Recall that for $0<h \leq 1$ an approximative sequence $\left(E^{h, i}\right)_{i=0}^{\infty}$ is defined recursively by setting first $E^{h, 0}=E_{0}$ and for each $i=0,1,2, \ldots$ the set $E^{h, i+1}$ is chosen to be a minimizer of $\mathcal{F}_{h, \bar{f}(h, i)}\left(\cdot, E^{h, i}\right)$ defined in (3.4), where $\bar{f}(h, i)=f_{i h}^{(i+1) h} f(t) \mathrm{d} t$. Recall also that $f:[0, \infty) \rightarrow \mathbb{R}$ is measurable and satisfies (3.1) (and hence $|\bar{f}(h, i)| \leq C_{0}$ ). Now each $E^{h, i}$, with $i \geq 1$, satisfies the Euler-Lagrange equation (3.6) with the constant $\Lambda=\bar{f}(h, i-1)$. Again, the corresponding approximative flat flow $\left(E^{h}\right)_{t \geq 0}$ is given by (3.3).

Our aim is to show that for a time interval $(0, \delta]$, with $\delta$ small enough, we may construct barrier sets $G^{h, i} \subset E^{h, i}$ for $i=1, \ldots,\lfloor\delta / h\rfloor+1$ such that for every $h \leq t \leq \delta$ the barrier $G^{h,\lfloor t / h\rfloor}$ contains a simply connected set $A_{t}$ defined as

$$
A_{t}=\mathbf{C}\left(c_{1} t, r\right) \cup B\left(-r e_{1}, r-c_{2} t\right) \cup B\left(r e_{1}, r-c_{2} t\right),
$$

with some $c_{1}, c_{2} \in \mathbb{R}_{+}$, depending only on $n, r$ and $C_{0}$, provided that $h$ is small enough. Now, if $\left(E_{t}\right)_{t \geq 0}$ is any cluster flow, then $A_{t} \subset E_{t}^{h}$ implies $\left|A_{t} \backslash E_{t}\right|=0$ for every $t \in(0, \delta)$ and further, since $E_{t} \in X_{n}$, this means $\operatorname{int}\left(A_{t}\right) \subset E_{t}$. The rest of the claim follows trivially from this.

We first note that it is easy to see that the balls $B\left( \pm r e_{1}, r-c_{2} t\right)$ are contained in $E_{t}$. Indeed, by possibly replacing $C_{0}$ with $\max \left\{C_{0}, 4(n-1) / r\right\}$, we may assume that $r / 4 \geq(n-1) / C_{0}$. Then by (i) of Proposition 3.2 and Proposition 3.3 we find $\eta=\eta\left(n, r / 2, C_{0}\right) \in \mathbb{R}_{+}$and $0<h_{0}=$ $h_{0}\left(n, r / 2, C_{0}\right) \leq 1$ such that for every $0<h \leq h_{0}$ the following implication holds

$$
B(x, \tilde{r}) \subset E^{h, i} \text { with } \tilde{r} \geq r / 2 \Longrightarrow \bar{B}(x, \tilde{r}-\eta h) \subset E^{h, i+1} .
$$

We split the proof into three steps.

Step 1: We prove that there is a positive $\alpha=\alpha\left(n, r, C_{0}\right)$, such that the set $E^{h, 1}$ contains the cylinder $\mathbf{C}\left(\alpha h^{\frac{1}{4}}, \alpha h^{\frac{1}{2}}\right)$ provided that $h$ is sufficiently small.

To this aim, let $\tau>0$ be a small number, which we will fix later. We use $C$ and $c$ for positive constants which may change from line to line but always depend only on $n, r$ and $C_{0}$. We also use a further shorthand notation $\mathbf{C}_{h, \tau}$ for the cylinder $\mathbf{C}\left(\tau h^{\frac{1}{4}}, \tau h^{\frac{1}{2}}\right)$ 
By (4.2) the balls $\bar{B}\left( \pm r e_{1}, r-\eta h\right)$ are contained in $E^{h, 1}$ provided that $h$ is small enough. Again, assuming $\tau \leq r / 2$ and $h$ to be sufficiently small we have

$$
\begin{aligned}
(r-\eta h)^{2}-\left(r-\tau h^{\frac{1}{2}}\right)^{2} & =\left(2 r-\eta h-\tau h^{\frac{1}{2}}\right)\left(\tau-\eta h^{\frac{1}{2}}\right) h^{\frac{1}{2}} \\
& >\frac{\tau r}{2} h^{\frac{1}{2}} \geq \tau^{2} h^{\frac{1}{2}} .
\end{aligned}
$$

Thus the heads of the cylinder $\mathbf{C}_{h, \tau}$, which are the vertical parts of the boundary, are contained in $B\left( \pm r e_{1}, r-\eta h\right)$ and therefore, in turn, in the set $E^{h, 1}$. Since $\partial E^{h, 1}$ is $C^{2}$ (possibly up to a closed singular part of Hausdorff-dimension at most $n-8)$, then by a foliation and continuity argument we may assume that $\mathcal{H}^{n-1}\left(\partial \mathbf{C}_{h, \tau} \cap \partial E^{h, 1}\right)=0$. Otherwise, we would choose $\tau / 2 \leq \tilde{\tau}<\tau$ such that the heads of the cylinder $\mathbf{C}_{h, \tilde{\tau}}$ are contained in $B\left( \pm r e_{1}, r-\eta h\right)$ and $\mathcal{H}^{n-1}\left(\partial \mathbf{C}_{h, \tilde{\tau}} \cap \partial E^{h, 1}\right)=0$. This implies

$$
P\left(\mathbf{C}_{h, \tau} \cup E^{h, 1}\right)=\mathcal{H}^{n-1}\left(\partial \mathbf{C}_{h, \tau} \backslash E^{h, 1}\right)+\mathcal{H}^{n-1}\left(\partial E^{h, 1} \backslash \mathbf{C}_{h, \tau}\right) .
$$

Again, we have the following estimates

$$
\begin{aligned}
\mathcal{H}^{n-1}\left(\partial \mathbf{C}_{h, \tau} \backslash E^{h, 1}\right) & \leq C \tau^{n-1} h^{\frac{n}{4}}, \\
\bar{d}_{E_{0}} & \leq C \tau^{2} h^{\frac{1}{2}} \text { in } \mathbf{C}_{h, \tau} \text { and } \\
\left|\mathbf{C}_{h, \tau}\right| & \leq C \tau^{n} h^{\frac{n+1}{4}} .
\end{aligned}
$$

We show that $\mathbf{C}\left(\tau h^{\frac{1}{4}} / 2, \tau h^{\frac{1}{2}}\right) \subset E^{h, 1}$ which implies the claim of Step 1 by choosing $\alpha=\tau / 2$. Suppose by contradiction that this does not hold. We first notice that $E_{0}=\mathbf{C}(g,[-2 r, 2 r])$ with a continuous $g$ having the zero value at the endpoints and therefore by Proposition 3.4 it holds $E^{h, 1}=\left(E^{h, 1}\right)^{*}$, i.e., $E^{h, 1}$ Schwarz symmetric with respect to $x_{1}$-axis. Using this and the fact that the heads of $\mathbf{C}_{h, \tau}$ are in $E^{h, 1}$, we conclude

$$
\mathcal{H}^{n-1}\left(\partial E^{h, 1} \cap \mathbf{C}_{h, \tau}\right) \geq c \tau^{n-1} h^{\frac{n-1}{4}} .
$$

We use the set $\mathbf{C}_{h, \tau} \cup E^{h, 1}$ as a competitor in the energy $\mathcal{F}_{h, \bar{f}(h, 0)}\left(\cdot, E_{0}\right)$. By using the previous estimate as well as (4.3), (4.4), (4.5) and (4.6) and assuming $h$ to be small enough we estimate

$$
\begin{aligned}
\mathcal{F}_{h, \bar{f}(h, 0)}\left(\mathbf{C}_{h, \tau} \cup E^{h, 1}, E_{0}\right) & =\mathcal{F}_{h, \bar{f}(h, 0)}\left(E^{h, 1}, E_{0}\right)+\mathcal{H}^{n-1}\left(\partial \mathbf{C}_{h, \tau} \backslash E^{h, 1}\right)-\mathcal{H}^{n-1}\left(\partial E^{h, 1} \cap \mathbf{C}_{h, \tau}\right) \\
& +\frac{1}{h} \int_{\mathbf{C}_{h, \tau} \backslash E^{h, 1}} \bar{d}_{E_{0}} \mathrm{~d} x-\bar{f}(h, 0)\left|\mathbf{C}_{h, \tau} \backslash E^{h, 1}\right| \\
& \leq \mathcal{F}_{h, \bar{f}(h, 0)}\left(E^{h, 1}, E_{0}\right)+C \tau^{n-1} h^{\frac{n}{4}}-c \tau^{n-1} h^{\frac{n-1}{4}} \\
& +C\left(\tau^{2} h^{-\frac{1}{2}}+1\right)\left|\mathbf{C}_{h, \tau}\right| \\
& \leq \mathcal{F}_{h, \bar{f}(h, 0)}\left(E^{h, 1}, E_{0}\right)+C \tau^{n-1} h^{\frac{n}{4}}-c \tau^{n-1} h^{\frac{n-1}{4}}+C \tau^{n+2} h^{\frac{n-1}{4}} \\
& \leq \mathcal{F}_{h, \bar{f}(h, 0)}\left(E^{h, 1}, E_{0}\right)+\frac{c}{2} \tau^{n-1} h^{\frac{n-1}{4}}-c \tau^{n-1} h^{\frac{n-1}{4}}+C \tau^{n+2} h^{\frac{n-1}{4}} \\
& =\mathcal{F}_{h, \bar{f}(h, 0)}\left(E^{h, 1}, E_{0}\right)+\tau^{n-1} h^{\frac{n-1}{4}}\left(C \tau^{2}-\frac{c}{2}\right) .
\end{aligned}
$$

Thus, by choosing $\tau<\sqrt{c /(2 C)}$ we have $\mathcal{F}_{h, \bar{f}(h, 0)}\left(\mathbf{C}_{h, \tau} \cup E^{h, 1}, E_{0}\right)<\mathcal{F}_{h, \bar{f}(h, 0)}\left(E^{h, 1}, E_{0}\right)$ which contradicts the minimality of the set $E^{h, 1}$. Hence, we have $\mathbf{C}\left(\alpha h^{\frac{1}{4}}, \alpha h^{\frac{1}{2}}\right) \subset E^{h, 1}$ for $\alpha=\tau / 2$. 
Step 2: We proceed by constructing candidate family for the barrier sets $G^{h, i}$, for every $i=1, \ldots,\lfloor\delta / h\rfloor+1$ and small $\delta$, which satisfy for every $h \leq t \leq \delta$ the condition $A_{t} \subset G^{h,\lfloor t / h\rfloor}$, where $A_{t}$ is defined in (4.1). To be more precise, we will define positive numbers $d_{h, i}, l_{h, i}$ and $r_{h, i}$ (such that $l_{h, i}$ increases and $r_{h, i}$ decreases linearly in discrete time and $r_{h, 1} \rightarrow r$ as $h \rightarrow 0$ ) and suitable convex and positive functions $\varphi_{h, i}:\left[-d_{h, i}, d_{h, i}\right] \rightarrow \mathbb{R}$ with $l_{h, i} / 2 \leq \varphi_{h, i} \leq l_{h, i}$. Then we define the barrier sets $G^{h, i}$, see Figure 4 , as the union

$$
G^{h, i}=\mathbf{C}\left(\varphi_{h, i},\left[-d_{h, i}, d_{h, i}\right]\right) \cup \bar{B}\left(-r e_{1}, r_{h, i}\right) \cup \bar{B}\left(r e_{1}, r_{h, i}\right) .
$$

Here it follows from the selection of the parameters and the functions that the heads of the

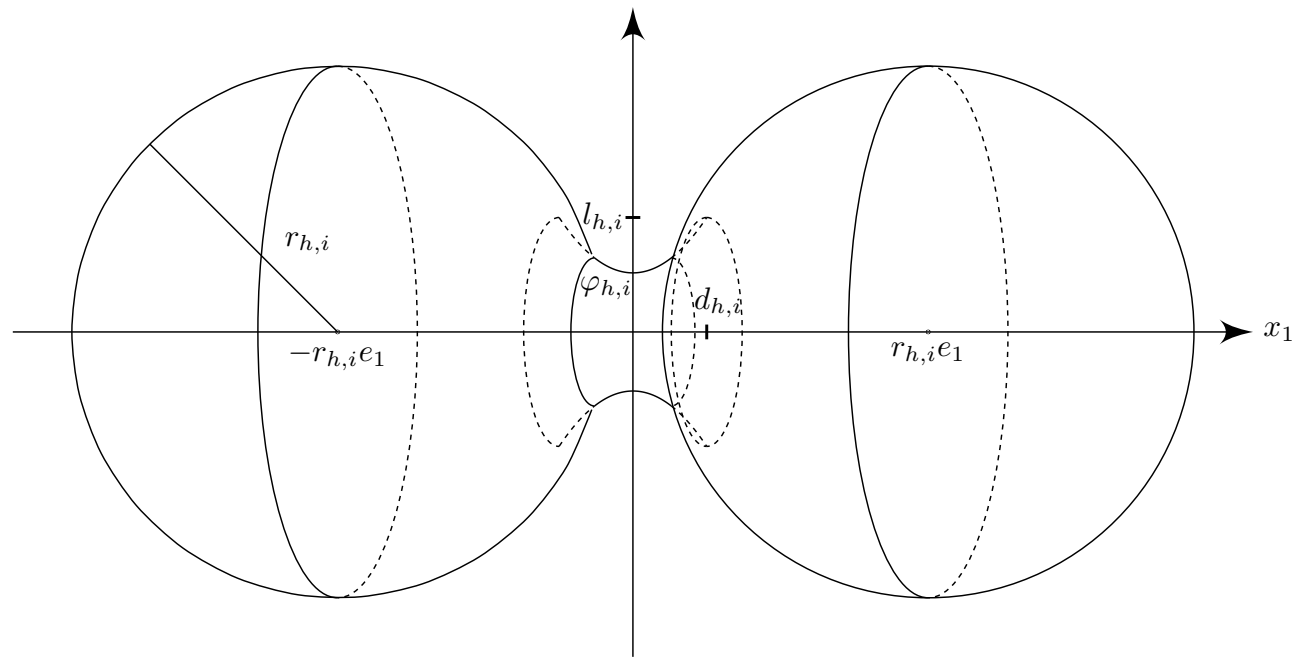

Figure 4.1. A visualization of the barrier set $G^{h, i}$.

neck $\mathbf{C}\left(\varphi_{h, i},\left[-d_{h, i}, d_{h, i}\right]\right)$ are contained in the balls $B\left( \pm r e_{1}, r_{h, i}\right)$ so $G^{h, i}$ will contain a simply connected set

$$
\mathbf{C}\left(l_{h, i} / 2, r\right) \cup \bar{B}\left(-r e_{1}, r_{h, i}\right) \cup \bar{B}\left(r e_{1}, r_{h, i}\right) .
$$

and hence the behavior of $l_{h, i}$ and $r_{h, i}$ yield the condition $A_{t} \subset G^{h,\lfloor t / h\rfloor}$.

To this end, let $0<\delta<1$ be a sufficiently small number which will ultimately depend only on $n, r$ and $C_{0}$. Note that it holds $h i \leq 2 \delta$ for all $i=1, \ldots,\lfloor\delta / h\rfloor+1$ when $h$ is small. We begin by setting $r_{h, i}=r-\eta h i$. Now $r_{h, i} \geq r-2 \eta \delta$ so by assuming $\delta$ to be small enough we have $r_{h, i} \geq r / 2$ and hence thanks to (4.2)

$$
\bar{B}\left(-r e_{1}, r_{h, i}\right) \cup \bar{B}\left(r e_{1}, r_{h, i}\right) \subset E^{h, i} .
$$

Again, set $\Lambda_{0}=\max \left\{4 \eta^{2}, 2^{9}(n-2)^{2}, 1\right\}$ and for each $i=1, \ldots,\lfloor\delta / h\rfloor+1$ define

$$
l_{h, i}=\Lambda_{0} h(i-1)+\alpha h^{\frac{1}{4}} \text { and } d_{h, i}=2 \eta h(i-1)+\alpha h^{\frac{1}{2}} .
$$

It follows from the choice of $\Lambda_{0}$ that for $\delta$ small enough $\left(\delta \leq \Lambda_{0}^{-2}\right)$ it holds

$$
\Lambda_{0}^{\frac{1}{2}} d_{h, i} \leq l_{h, i} .
$$

Moreover, $l_{h, i} \leq \Lambda_{0} \delta+\alpha \delta^{\frac{1}{4}}$ so by decreasing $\delta$ we may assume that $d_{h, i}$ and $l_{h, i}$ are as small as we need. Note that by Step 1 we have

$$
\mathbf{C}\left(l_{h, 1}, d_{h, 1}\right) \subset E^{h, 1} .
$$


Further, by replacing $\alpha$ with $\min \{\alpha, r / 4\}$, if necessary, we have

$$
\begin{aligned}
r_{h, i}^{2}-\left(r-d_{h, i}\right)^{2} & =\left(r_{h, i}+r-d_{h, i}\right)\left(r_{h, i}-r+d_{h, i}\right) \\
& =\left(2 r-\eta h(3 i-2)-\alpha h^{\frac{1}{2}}\right)\left(\eta h(i-2)+\alpha h^{\frac{1}{2}}\right) \\
& \geq r\left(\eta h(i-2)+\alpha h^{\frac{1}{2}}\right) \\
& =r \eta h(i-1)+r\left(\alpha-\eta h^{\frac{1}{2}}\right) h^{\frac{1}{2}} \\
& \geq r \eta h(i-1)+\frac{\alpha r}{2} h^{\frac{1}{2}} \\
& \geq 2 \delta \Lambda_{0}^{2} h(i-1)+2 \alpha^{2} h^{\frac{1}{2}} \\
& \geq 2 \Lambda_{0}^{2} h^{2}(i-1)^{2}+2 \alpha^{2} h^{\frac{1}{2}} \\
& \geq\left(\Lambda_{0} h(i-1)+\alpha h^{\frac{1}{4}}\right)^{2}=l_{h, i}^{2},
\end{aligned}
$$

when $\delta$ is small. Therefore, by Pythagorean theorem

$$
\left\{\left( \pm d_{h, i}, x^{\prime}\right) \in \mathbb{R}^{n}: x^{\prime} \in \bar{B}^{n-1}\left(0, l_{h, i}\right)\right\} \subset \bar{B}\left(-r e_{1}, r_{h, i}\right) \cup \bar{B}\left(r e_{1}, r_{h, i}\right),
$$

i.e., the heads of the cylinder $\mathbf{C}\left(l_{h, i}, d_{h, i}\right)$ are contained in the balls $\bar{B}\left( \pm r e_{1}, r_{h, i}\right)$.

We define for each $i=1, \ldots,\lfloor\delta / h\rfloor+1$ convex function $\varphi_{h, i}:\left[-d_{h, i+1}, d_{h, i+1}\right] \rightarrow \mathbb{R}$ by setting

$$
\varphi_{h, i}(t)=\frac{a_{h, i}}{2}\left(t^{2}-d_{h, i}^{2}\right)+l_{h, i},
$$

where $a_{h, i}=\Lambda_{0}^{\frac{1}{2}} / l_{h, i}$. Note that by (4.9) we have $a_{h, i} d_{h, i} \leq 1$ and further $a_{h, i} d_{h, i}^{2} \leq l_{h, i}$. Thus, $\varphi_{h, i}$ is 1-Lipschitz and

$$
\varphi_{h, i} \geq \varphi_{h, i}(0)=l_{h, i}-\frac{1}{2} a_{h, i} d_{h, i}^{2} \geq \frac{l_{h, i}}{2} .
$$

Recall, that we set $G^{h, i}$ as the union

$$
G^{h, i}=\mathbf{C}\left(\varphi_{h, i},\left[-d_{h, i}, d_{h, i}\right]\right) \cup \bar{B}\left(-r e_{1}, r_{h, i}\right) \cup \bar{B}\left(r e_{1}, r_{h, i}\right) .
$$

By (4.11) and (4.12) the barrier $G^{h, i}$ contains the simply-connected set

$$
\mathbf{C}\left(l_{h, i} / 2, r\right) \cup B\left(-r e_{1}, r_{h, i}\right) \cup B\left(r e_{1}, r_{h, i}\right)
$$

and thus recalling $l_{h, i}=\Lambda_{0} h(i-1)+\alpha h^{\frac{1}{4}}$ and $r_{h, i}=r-\eta h i$ we find $c_{1}, c_{2} \in \mathbb{R}_{+}$, depending only on $n, r$ and $C_{0}$, such that for every $h \leq t \leq \delta$ the barrier $G^{h, \mid t / h\rfloor}$ contains the set $A_{t}$ defined in (4.1) with the constants $c_{1}, c_{2}$.

Step 3: We finish the proof by showing that each barrier $G^{h, i}$ constructed in the previous step is actually contained in $E^{h, i}$. First, we conclude from (4.8), (4.9) and $\Lambda_{0} \geq 2 \eta$ that when $\delta$ is small enough then for every $i=2, \ldots,\lfloor\delta / h\rfloor+1$ it holds

$$
\left|\varphi_{h, i}-\varphi_{h, i-1}\right| \leq 2 \Lambda_{0} h \text { on }\left[-d_{h, i-1}, d_{h, i-1}\right] .
$$

Further, using the fact that $\varphi_{h, i}$ is 1-Lipschitz and $r_{h, i-1} \geq r_{h, i}$ we obtain

$$
G^{h, i} \subset\left\{x \in \mathbb{R}^{n}: \bar{d}_{G^{h, i-1}}(x) \leq 4 \Lambda_{0} h\right\} .
$$


By (4.7) and (4.10) we have $G^{h, 1} \subset E^{h, 1}$. Thus, we argue by induction. Assume that for $i=2, \ldots,\lfloor\delta / h\rfloor+1$ it holds $G^{h, i-1} \subset E^{h, i-1}$. By (4.8) and (4.12) we have for small $\delta$

$$
\varphi_{h, i}-2 \Lambda_{0} h \geq \frac{l_{h, i}}{2}-2 \Lambda_{0} h \geq \frac{\alpha}{2} h^{\frac{1}{4}}-2 \Lambda_{0} h>0
$$

and hence the set $\mathbf{C}\left(\varphi_{h, i}-2 \Lambda_{0} h,\left[-d_{h, i}, d_{h, i}\right]\right)$ is well-defined. Again, by (4.13) and $\varphi_{h, i}-2 \Lambda_{0} h \leq$ $l_{h, i-1}$ it holds

$$
\mathbf{C}\left(\varphi_{h, i}-\Lambda_{0} h,\left[-d_{h, i}, d_{h, i}\right]\right) \subset G^{h, i-1} .
$$

Next, we define an auxiliary set $\tilde{G}^{h, i} \subset G^{h, i}$ as

$$
\tilde{G}^{h, i}=\mathbf{C}\left(\varphi_{h, i}-2 \Lambda_{0} h,\left[-d_{h, i}, d_{h, i}\right]\right) \cup \bar{B}\left(-r e_{1}, r_{h, i}\right) \cup \bar{B}\left(r e_{1}, r_{h, i}\right) .
$$

Then by the induction assumption, (4.15) and $r_{h, i-1} \geq r_{h, i}$ we have $\tilde{G}^{h, i} \subset E^{h, i-1}$ and therefore by $(3.5)$

$$
\left\{x \in \tilde{G}^{h, i}: \operatorname{dist}\left(x, \partial \tilde{G}^{h, i}\right)>\gamma h^{\frac{1}{2}}\right\} \subset E^{h, i} .
$$

Since the function $t \mapsto \max \left\{s:\{t\} \times \bar{B}^{n-1}(0, s) \subset \tilde{G}^{h, i}\right\}$ is increasing in $[0, r]$, decreasing in $[-r, 0]$ and $\tilde{G}^{h, i}$ is a solid of revolution, then for any

$$
x \in \mathbf{C}\left(\varphi_{h, i}-2 \Lambda_{0} h,\left[-d_{h, i}, d_{h, i}\right]\right) \backslash\left(\bar{B}\left(-r e_{1}, r_{h, i}\right) \cup \bar{B}\left(r e_{1}, r_{h, i}\right)\right)
$$

the closest point $y \in \partial \tilde{G}^{h, i}$, i.e. $\operatorname{dist}\left(x, \partial \tilde{G}^{h, i}\right)=|x-y|$, must lie on

$$
\partial \mathbf{C}\left(\varphi_{h, i}-2 \Lambda_{0} h,\left[-d_{h, i}, d_{h, i}\right]\right) \backslash\left(\bar{B}\left(-r e_{1}, r_{h, i}\right) \cup \bar{B}\left(r e_{1}, r_{h, i}\right)\right) .
$$

Let us write $x=\left(x_{1}, x^{\prime}\right)$ and $y=\left(y_{1}, y^{\prime}\right)$. Since $\varphi_{h, i}$ is a 1-Lipschitz function, then

$$
\begin{aligned}
\left|\varphi_{h, i}\left(x_{1}\right)-\right| x^{\prime}|| & \leq\left|\varphi_{h, i}\left(x_{1}\right)-\varphi_{h, i}\left(y_{1}\right)\right|+\left|\varphi_{h, i}\left(y_{1}\right)-\right| x^{\prime}|| \\
& \leq\left|x_{1}-y_{1}\right|+\| y^{\prime}|-| x^{\prime}|| \\
& \leq 2|x-y|=2 \operatorname{dist}\left(x, \partial \tilde{G}^{h, i}\right) .
\end{aligned}
$$

We have $\gamma h^{\frac{1}{2}}>2 \Lambda_{0} h$ and $\alpha h^{\frac{1}{4}}>12 \gamma h^{\frac{1}{2}}$, provided that $\delta$ is small, and thus by (4.8) and (4.12)

$$
\frac{l_{h, i}}{4} \leq \varphi_{h, i}-3 \gamma h^{\frac{1}{2}}<\varphi_{h, i}-2 \Lambda_{0} h-2 \gamma h^{\frac{1}{2}} \text { on }\left[-d_{h, i}, d_{h, i}\right] .
$$

Therefore, it follows from (4.7), (4.16), (4.17) and (4.18) that the set $\mathbf{C}\left(\varphi_{h, i}-3 \gamma h^{\frac{1}{2}},\left[-d_{h, i}, d_{h, i}\right]\right)$ is well-defined and contained in $E^{h, i}$.

We argue by contradiction and assume that $G^{h, i}$ is not contained in $E^{h, i}$. Since

$$
\mathbf{C}\left(\varphi_{h, i}-3 \gamma h^{\frac{1}{2}},\left[-d_{h, i}, d_{h, i}\right]\right) \subset E^{h, i},
$$

we may lift up the graph of $\varphi_{h, i}-3 \gamma h^{\frac{1}{2}}$ until it touches the boundary $\partial E^{h, i}$. To be more precise, by a continuity argument and (4.7) there is $0<\tau<3 \gamma h^{\frac{1}{2}}$ such that

$$
\mathbf{C}\left(\varphi_{h, i}-\tau,\left[-d_{h, i}, d_{h, i}\right]\right) \subset E^{h, i}
$$

and there is a point $z \in \Gamma \cap \partial E^{h, i}$, where

$$
\Gamma=\left\{\left(x_{1}, x^{\prime}\right) \in \mathbb{R}^{n}: x_{1} \in\left(-d_{h, i}, d_{h, i}\right), x^{\prime} \in \partial B^{n-1}\left(0, \varphi_{h, i}\left(x_{1}\right)-\tau\right)\right\} .
$$


In particular, the boundary $\partial E^{h, i}$ satisfies interior ball condition at $z$ and thus $z$ belongs to the regular part of $\partial E^{h, i}$. Hence, by the comparison principle we have $H_{E^{h, i}}(z) \leq H_{\Gamma}(z)$, where $H_{\Gamma}$ is chosen to be compatible with the inside-out orientation of

$$
\mathbf{C}\left(\varphi_{h, i}-\tau,\left[-d_{h, i}, d_{h, i}\right]\right) \text {. }
$$

Recalling (2.2), (4.18), $a_{h, i} d_{h, i} \leq 1$ and the choice of $\Lambda_{0}$ we estimate

$$
\begin{aligned}
H_{\Gamma}(z) & =-\frac{\varphi_{h, i}^{\prime \prime}\left(z_{1}\right)}{\left(1+\left(\varphi_{h, i}^{\prime}\left(z_{1}\right)\right)^{2}\right)^{\frac{3}{2}}}+\frac{1}{\left(1+\left(\varphi_{h, i}^{\prime}\left(z_{1}\right)\right)^{2}\right)^{\frac{1}{2}}} \frac{(n-2)}{\varphi_{h, i}\left(z_{1}\right)-\tau} \\
& =-\frac{a_{h, i}}{\left(1+\left(a_{h, i} z_{1}\right)^{2}\right)^{\frac{3}{2}}}+\frac{1}{\left(1+\left(a_{h, i} z_{1}\right)^{2}\right)^{\frac{1}{2}}} \frac{(n-2)}{\varphi_{h, i}\left(z_{1}\right)-\tau} \\
& \leq-\frac{a_{h, i}}{2^{\frac{3}{2}}}+\frac{4(n-2)}{l_{h, i}} \\
& =\frac{2^{\frac{7}{2}}(n-2)-\Lambda_{0}^{\frac{1}{2}}}{2^{\frac{3}{2}} l_{h, i}} \leq-\frac{\Lambda_{0}^{\frac{1}{2}}}{2^{\frac{5}{2}} l_{h, i}} .
\end{aligned}
$$

Thus, by choosing $\delta$ to be small enough we have $H_{\Gamma}(z) \leq-\left(5 \Lambda_{0}+C_{0}\right)$. Then the EulerLangrange equation (3.6) for $E^{h, i}$ and $H_{E^{h, i}}(z) \leq H_{\Gamma}(z)$ yield

$$
\bar{d}_{G^{h, i-1}}(z)=-H_{E^{h, i}}(z) h+\bar{f}(h, i-1) h \geq 5 \Lambda_{0} h .
$$

However, by the construction we have $z \in G^{h, i}$ and thus the above contradicts (4.14). Hence, we have $G^{h, i} \subset E^{h, i}$ for every $i=1, \ldots,\lfloor\delta / h\rfloor+1$.

\section{ACKNOWLEDGMENTS}

The research was supported by the Academy of Finland grant 314227 .

\section{REFERENCES}

[1] F. Almgren, J.E. Taylor, L. Wang, Curvature-driven flows: a variational approach. SIAM J. Control Optim. 31(2), 387-438 (1993).

[2] M. Barchiesi, F. Cagnetti, N. Fusco, Stability of the Steiner symmetrization of convex sets. J. Eur. Math. Soc., 15 (2013), pp. 1245-1278.

[3] G. Bellettini, Lecture notes on mean curvature flow: barriers and singular perturbations. Lecture Notes (Scuola Normale Superiore) vol. 12, Edizioni della Normale, Pisa (2013).

[4] G. Bellettini, M. Paolini, Some results on minimal barriers in the sense of De Giorgi applied to driven motion by mean curvature. Rend. Accad. Naz. Sci. XL Mem. Mat. Appl. (5) 19 (1995), 43-67. Errata, ibid. 26 (2002), 161-165.

[5] K.A. Brakke, The Motion of a Surface by its Mean Curvature. Math. Notes 20, Princeton Univ. Press, Princeton, NJ (1978).

[6] A. Chambolle, M. Morini, M. Ponsiglione, Nonlocal curvature flows, Arch. Ration. Mech. Anal. 12 (2015), 1263-1329

[7] Y.G. Chen, Y. Giga, S. Goto, Uniqueness and existence of viscosity solutions of generalized mean curvature. Proc. Japan Acad. Ser. A Math. Sci. 65 (1989), 207-210.

[8] M. Delgadino, F. Maggi, Alexandrov's theorem revisited. Anal. PDE 12 (2019), 1613-1642.

[9] N. DirR, S. Luckhaus, M. Novaga. A stochastic selection principle in case of fattening for curvature flow. Calc. Var. Partial Differential Equations 4 (2001), 405-425.

[10] L.C. Evans, J. Spruck, Motion of level sets by mean curvature I. J. Differential Geom. 33 (1991), $635-681$.

[11] N. Fusco, V. Julin, M. Morini, Stationary sets and asymptotic behavior of the mean curvature flow with forcing in the plane, Preprint 2020. https://arxiv.org/abs/2004.07734 
[12] M. A. Grayson, A short note on the evolution of a surface by its mean curvature. Duke Math. J. 58 (1989), 555-558.

[13] R. Gulliver, Y. Koo, Sharp growth rate for generalized solutions evolving by mean curvature plus a forcing term. J. Reine Angew. Math. 538 (2001), 1-24.

[14] S. Luckhaus, T. Stürzenhecker, Implicit time discretization for the mean curvature flow equation. Calc. Var. Partial Differential Equations 3 (1995), 253-271.

[15] F. MagGi, Sets of finite perimeter and geometric variational problems. An introduction to geometric measure theory. Cambridge Studies in Advanced Mathematics, 135. Cambridge University Press, Cambridge (2012).

[16] L. Mugnai, C. Seis, E. Spadaro, Global solutions to the volume-preserving mean-curvature flow. Calc. Var. Partial. Diff. Eq. 55 (2016), Art. 18, 23 pp. 